\title{
USO DA SIMULAÇÃO DE MONTE CARLO EM PROJETOS DE CONSTRUÇÃO DE RODOVIAS NO NORTE FLUMINENSE
}

\author{
Aníbal Alberto Ignácio Vilcapoma \\ UFF/PURO \\ Rua Recife, s/n - Jardim Bela Vista - Rio das Ostras - RJ \\ avilcap@vm.uff.br \\ Luize de Magalhães Moura \\ UFF/PURO \\ Rua Recife, s/n - Jardim Bela Vista - Rio das Ostras - RJ \\ luizemoura@gmail.com \\ Léa Maria Dantas Sampaio \\ COPPE/UFRJ \\ Centro de Tecnologia - Cidade Universitária - \\ I. do Fundão - Rio de Janeiro - RJ \\ leasampaio@gmail.com
}

\begin{abstract}
Resumo
Para se projetar e se dimensionar a construção de estradas há que se considerar muitos aspectos estocásticos. Falhas no dimensionamento de projetos de engenharia causam atrasos, multas e custos elevados para as empresas envolvidas. O presente artigo trata do uso da simulação de monte Carlo na avaliação da duração e dos custos, considerados em projetos de construção de rodovias, em cidade do Norte Fluminense.
\end{abstract}

Palavras chaves: $\quad$ Simulação de monte Carlo, construção de estrada.

\begin{abstract}
In order to project and sizing the construction of roads is necessary to consider many stochastic aspects. Flaws in the design of engineering projects cause delays, penalties and high costs for the companies involved. The present article describes the use of Monte Carlo simulation in the evaluation of time and costs, considered in highway construction projects in cities of Norte Fluminense.

Keywords: $\quad$ Monte Carlo simulation, road construction.
\end{abstract}




\section{INTRODUÇAO}

A indústria da construção civil tem particularidades que a distingue das demais, refletindo em uma estrutura dinâmica e complexa, pois reúnem materiais, máquinas, equipamentos, conjunto de diversos tipos de profissionais, para os mais diversos tipos de realizações.

Neste artigo, procura-se realizar um estudo empírico relacionado à construção de uma rodovia em uma cidade impactadas pelos investimentos da Indústria do Petróleo e gás da Bacia de Campos. A instalação da Sede da Petrobrás tornou o município base de um grande aglomerado petrolífero, diante deste cenário, houve um crescimento acentuado no número de empregos gerados na cidade e região, o que ocasiona uma enorme migração, nesta cidade.

Com o crescimento gerado pela exploração de petróleo, a cidade deparou-se com problemas de infraestrutura para atender a crescente população, bem como o deslocamento e a mobilidade entre cidades próximas. Para minimizar esse ônus causado pelo desenvolvimento, as prefeituras propõem, como investimento, a construção de rodovias que interliguem essas cidades.

Neste contexto a pesquisa se torna relevante no dimensionamento e o tempo de duração dos trabalhos realizado. Simulações no que tange os custos e atividades envolvidas na construção da rodovia, através da elaboração da rede PERT/CPM e de simulações correspondentes às atividades e suas durações pelo método de Monte Carlo. Para o tratamento dos dados foi utilizado o software Microsoft Excel.

\section{REFERENCIAL TEÓRICO}

A indústria da construção civil difere muito das demais visto que apresenta peculiaridades que refletem sua estrutura dinâmica e complexa e que variam desde profissionais, equipamentos, máquinas, até condições climáticas ideais para que a obra seja realizada.

Trabalhos como o de Barra et al. (2013) apresentam o uso da elaboração de rede PERT/CPM na indústria da construção civil, através de estudos de caso. Os autores do trabalho apresentam a aplicação da ferramenta, através do software MS Project, no planejamento e gerenciamento da construção de uma escola.

Araújo e Meira (1997) mostram em seu trabalho o papel do planejamento interligado ao controle gerencial nas pequenas empresas de construção civil, onde mostram uso de diversas ferramentas incluindo a rede PERT/COM como formas de ajudar no planejamento e controle das obras.

Rodrigues e Soares (2005), em seu trabalho, utilizam a simulação de Monte Carlo no intuito de possibilitar ao empreendedor do ramo da construção civil, avaliar os riscos aos quais estará sujeito o empreendimento, sendo assim a tomada de decisões mais segura, resultando, desta forma, na otimização dos lucros. Para os autores, o crescimento e a sobrevivência das empresas de construção civil estão intimamente relacionados com a capacidade destas em estimarem seus custos de construção com a máxima precisão possível, isto é, com margem de erro mínima em relação aos custos apropriados ao longo da construção de seus empreendimentos, sendo a simulação de Monte Carlo uma ferramenta de aumento de previsão orçamentária na estimação do custo global (RODRIGUES E SOARES, 2005).

Mostra-se a seguir a revisão conceitual com as definições relativas a rede PERT/CPM e ao método de Monte Carlo, os quais serviram para dar embasamento teórico ao artigo.

\subsection{MODELO PERT/CPM}

O modelo PERT/CPM - Program Evaluation Review Technique/Critical Path Method (Técnica de Avaliação e Revisão de Programa/Método do Caminho Crítico), pode ser definido como um conjunto de processos e técnicas para planejamento, programação e controle de um empreendimento, operação ou projeto, tendo como característica fundamental 
a indicação, dentre as várias sequencias operacionais, daquela que possui duração máxima, além de permitir a indicação de graus de prioridade relativos, demonstrando distribuição de recursos e interdependência entre as várias ações necessárias ao desenvolvimento do projeto (CUKIERMAN,2000).

A rede PERT/COM também é definida como técnica de representação do plano de execução de um projeto por meio de um diagrama que mostra as interrelações entre as diversas atividades, além de informações relativas ao prazo (STONNER, 2000)

De acordo com Miranda e Almeida (2002) no modelo PERT/CPM, um projeto pode ser visualizado como um conjunto de operações conduzidas em uma sequencia para atingir dados objetivos. Identificadas as atividades, elas podem ser representadas e ordenadas em um Digrama de Rede que mostra as diversas sequencias de caminhos (atividades) e enfatiza o caminho crítico em função do tempo. Este caminho crítico é o que tem a maior duração dentre todos os outros, e é ele quem governa a duração do projeto.

\subsection{Método de Monte Carlo}

O método de Monte Carlo é uma técnica de amostragem que faz uso de variáveis aleatórias e distribuição de probabilidades para definir o comportamento do sistema em estudo. De acordo com Donatelli e Konrath (2005), quando o modelo envolve amostragem aleatória de uma variável em estudo, através de sua distribuição de probabilidade, este é chamado modelo de Simulação de Monte Carlo.

Segundo Martins et al (2010), as saídas de um processo de modelagem e simulação de Monte Carlo incluem itens como: (i) uma distribuição para cada variável de saída; (ii) uma listagem de sensibilidade das variáveis chaves ordenadas segundo sua correlação com a variável de saída; (iii) vários gráficos e resumos estatísticos que caracterizem os resultados simulados. Os autores ressaltam ainda que a simulação de Monte Carlo não fornece a tomada de decisão explicita como resultado, mas apresenta um detalhamento para a análise das possibilidades de resultado através de uma distribuição de frequência.

As bases para a aplicação da Simulação de Monte Carlo no cálculo da incerteza consistem em selecionar aleatoriamente um número de uma distribuição de possíveis valores para uma grandeza de entrada e repetir o procedimento para as outras grandezas de entrada (PIZZOLATO,2005).

\subsection{Distribuição Triangular}

A distribuição triangular é utilizada em situações em que não se conhece a forma exata da distribuição de probabilidade, mas se possui estimativas para o menor valor, o valor mais provável de ocorrer e o maio valor. A distribuição é assimétrica: à esquerda, quando o pico está fechado para o mínimo, e à direita, quando o pico está fechado ao máximo.

$$
f(x)=\left\{\begin{array}{cl}
\frac{2(x-a)}{(m-a)(b-a)}, & a \leq X \leq m \\
\frac{2(b-x)}{(b-m)(b-a)} & , m \leq X \leq b \\
0 & , X<a \text { ou } X>b
\end{array}\right.
$$

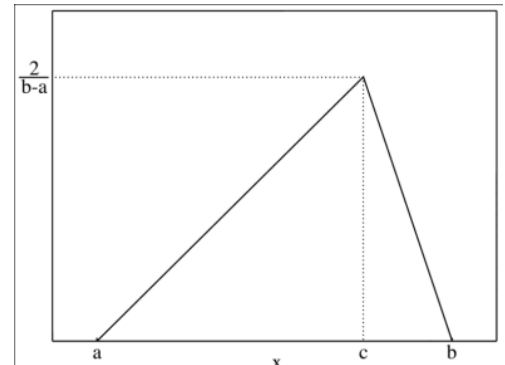

Figura 1- modelo matemático referente à distribuição triangular

\section{ESTUDO DE CASO}

O estudo tem como base a construção de uma rodovia a ser utilizada para diminuir o trânsito entre duas cidades, localizadas no Norte Fluminense. A construção normalmente é realizada de forma terceirizada, desde a contratação dos funcionários até dos equipamentos fiscais etc. 


\subsection{CARACTERÍSTICAS DA OBRA PESQUiSADA}

Os valores considerados nas atividades correspondentes à construção são estipulados, levando-se em conta obras existentes desse tipo, em diversas partes do estado do Rio de Janeiro. São valores fictícios, criados a partir de valores reais, em relação ao percentual atribuído a todo o projeto.

O pagamento da obra é através de medição do andamento físico da mesma junto ao órgão governamental responsável. A duração planejada para construção da rodovia é de 12 meses, não sendo consideradas eventuais multas pelo atraso, seu custo estimado é de $\mathrm{R} \$$ 14.560.000,00.

Considera-se, no presente artigo, o cronograma financeiro, conforme verificado em outros estudos. Neste cronograma, constam as atividades, seu custo em relação a cada mês, e seu valor (Tabela 1), sendo assim possível a elaboração de uma rede PERT/CPM conforme pode ser visto na Tabela 2.

Neste estudo, utiliza-se do Método de Monte Carlo com o intuito de prover simulações de prazos na entrega de cada atividade que compõe o projeto.

O Método de Monte Carlo, utilizado no modelo, gera milhares de iterações possíveis para cada uma das atividades, que leva em consideração as suas distribuições de tempo, gerando uma amostra. Tal amostra serve como fonte para verificação de gargalos que acontecem durante a construção da rodovia. Ainda de posse dessa consulta, pode-se utilizar o método para simular o quanto o custo relacionado pode variar de acordo com o tempo despendido em cada atividade.

\begin{tabular}{|c|c|c|}
\hline Atividade & Valor (mensal) & Tempo (meses) \\
\hline $\begin{array}{l}\text { Serviços de } \\
\text { acompanhamento }\end{array}$ & $\mathrm{R} \$ 128.128,00$ & 12 \\
\hline Transporte de equipamentos & $\mathrm{R} \$ 88.670,40$ & 2 \\
\hline Implantação de canteiro & $\mathrm{R} \$ 112.359,52$ & 2 \\
\hline Controle tecnológico & $\mathrm{R} \$ 8.109,92$ & 4 \\
\hline $\begin{array}{l}\text { Rebaixamento do lençol } \\
\text { freático }\end{array}$ & $\mathrm{R} \$ 319.009,60$ & 6 \\
\hline Estrada & $\mathrm{R} \$ 463.736,00$ & 5 \\
\hline $\begin{array}{l}\text { Estrada para rebaixamento } \\
\text { do lençol freático }\end{array}$ & $\mathrm{R} \$ 278.241,60$ & 1,5 \\
\hline $\begin{array}{l}\text { Estrada para construção de } \\
\text { rotatória }\end{array}$ & $\mathrm{R} \$ 185.494,40$ & 1,5 \\
\hline $\begin{array}{l}\text { Construção da primeira parte } \\
\text { da rotatória }\end{array}$ & $\mathrm{R} \$ 135.553,60$ & 1,5 \\
\hline $\begin{array}{l}\text { Construção da segunda parte } \\
\text { da rotatória }\end{array}$ & $\mathrm{R} \$ 135.553,60$ & 1,5 \\
\hline Via secundária 1 & $\mathrm{R} \$ 361.088,00$ & 3 \\
\hline Via secundária 2 e 3 & $\mathrm{R} \$ 436.800,00$ & 3 \\
\hline
\end{tabular}

Tabela 1 - Cronograma financeiro

Definição das atividades da obra

$\mathrm{Na}$ tabela a seguir, apresentar-se-ão as definições, de acordo com o engenheiros que atuam nesse tipo de construção, das atividades que compõe a obra conforme apresentado no cronograma.

\begin{tabular}{|c|c|c|}
\hline 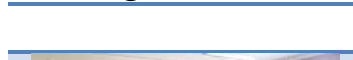 & Atividade & Definição \\
\hline Mा & Serviços de acompanhamento & $\begin{array}{l}\text { Contrato de serviços } \\
\text { independente da mão de obra } \\
\text { executado ao longo do projeto } \\
\text { de construção, sendo estes } \\
\text { como: aluguel de containers, } \\
\text { placas de obra, serviços } \\
\text { administrativos, dentre }\end{array}$ \\
\hline
\end{tabular}


outros.

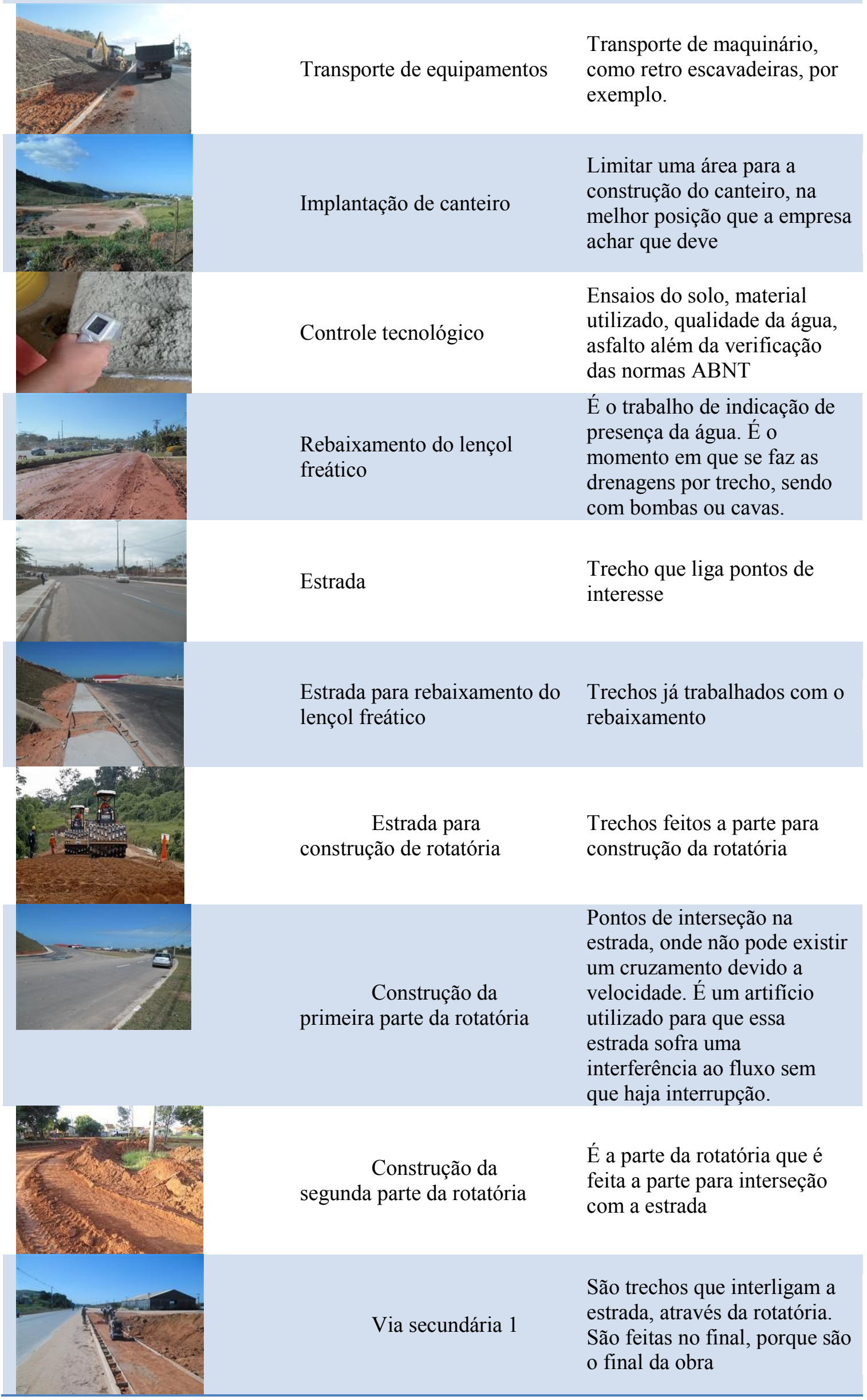


Vias paralelas a construção da estrada, que serão interligadas pela rotatória.

Tabela 2 - Definição das atividades

\subsection{ELABORAÇÃo dA REDE PERT/CPM}

A Tabela 3 contem os dados de cada atividade envolvida no planejamento, com suas descrições e as atividades com as quais se relacionam (precedentes), assim como o tempo de cada uma para, então, ser trabalhada a rede PERT/CPM.

\begin{tabular}{|c|c|c|c|}
\hline Atividade & Descrição & Precedência & $\begin{array}{c}\text { Tempo planejado } \\
\text { (meses) }\end{array}$ \\
\hline A & $\begin{array}{l}\text { Serviços de } \\
\text { acompanhamento }\end{array}$ & - & 12 \\
\hline B & $\begin{array}{l}\text { Transporte de } \\
\text { equipamentos }\end{array}$ & - & 2 \\
\hline $\mathrm{C}$ & $\begin{array}{l}\text { Implantação de } \\
\text { canteiro }\end{array}$ & - & 2 \\
\hline $\mathrm{D}$ & Controle tecnológico & B-C-E-F1 & 4 \\
\hline $\mathrm{E}$ & $\begin{array}{l}\text { Rebaixamento do } \\
\text { lençol freático }\end{array}$ & B-C & 6 \\
\hline $\mathrm{F}$ & Estrada & - & 5 \\
\hline F1 & $\begin{array}{l}\text { Estrada para } \\
\text { rebaixamento do } \\
\text { lençol freático }\end{array}$ & B-C-E & 1,5 \\
\hline $\mathrm{F} 2$ & $\begin{array}{l}\text { Estrada para } \\
\text { construção de } \\
\text { rotatória }\end{array}$ & B-C & 1,5 \\
\hline G1 & $\begin{array}{l}\text { Construção da } \\
\text { primeira parte da } \\
\text { rotatória }\end{array}$ & B-C-F2 & 1,5 \\
\hline G2 & $\begin{array}{l}\text { Construção da } \\
\text { segunda parte da } \\
\text { rotatória }\end{array}$ & B-C-F2- & 1,5 \\
\hline $\mathrm{H}$ & Via secundária 1 & B-C-F2- & 3 \\
\hline I & Via secundária 2 e 3 & G1-G2 ${ }^{\text {B-C-F2- }}$ & 3 \\
\hline
\end{tabular}

Tabela 3 - Tabela de precedência

De posse dos dados apresentados na tabela, construiu-se a rede PERT/CPM, conforme exposto na Figura 2. 


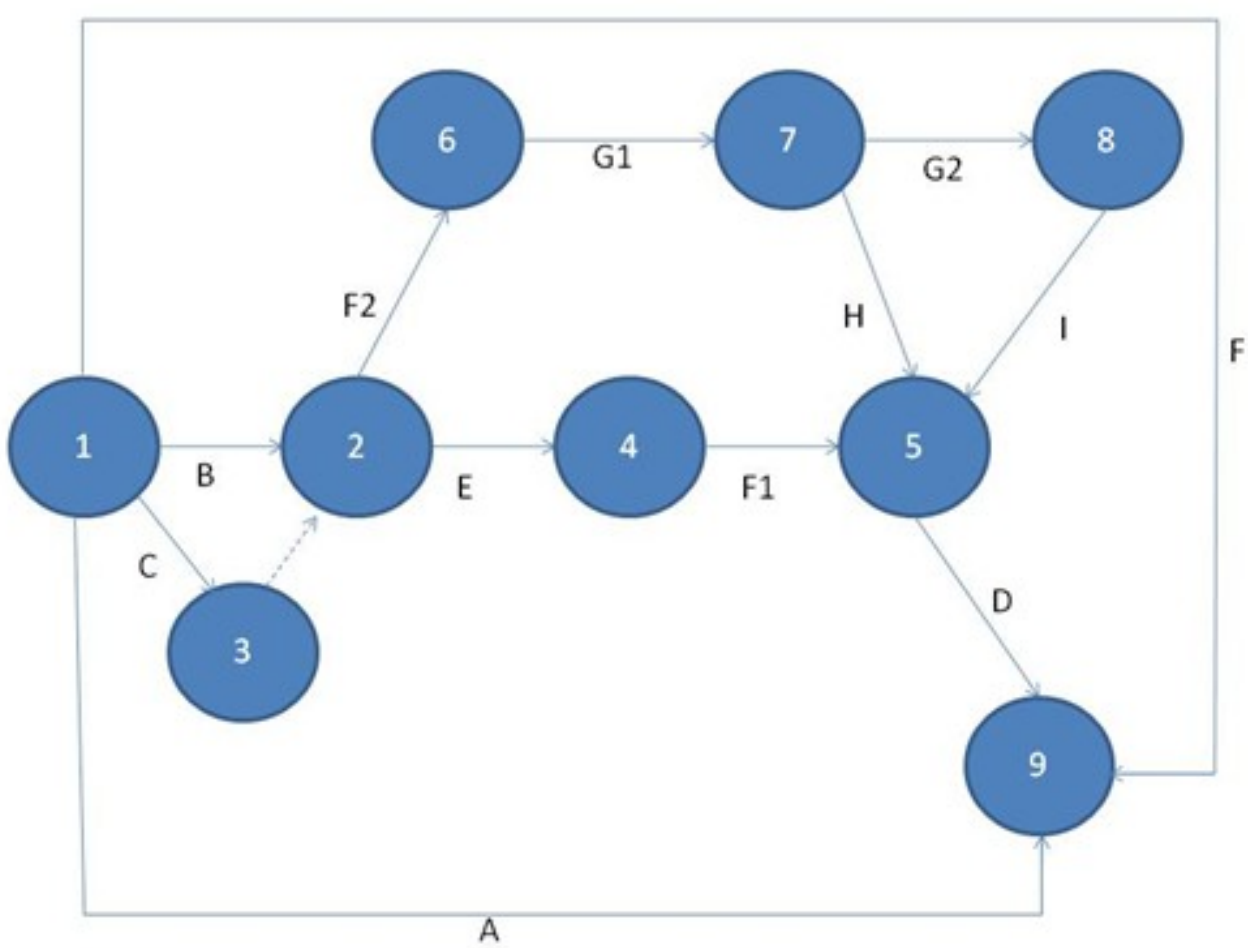

Figura 2 - Rede PERT/CPM

\subsection{ElaboraÇão do simulador, utilizando método de Monte Carlo.}

Com a construção da rede PERT/CPM foi possível verificar os caminhos para a construção da rodovia, sendo eles:

O caminho crítico identificado no modelo foi o CF2G1G2ID. Esses caminhos terão suas durações somadas no simulador, gerando o tempo total de cada um. Fazendo o uso dos números aleatórios será possível estimar a duração média da construção da rodovia.

Após a construção da rede PERT/CPM, criou-se um simulador, baseado no método de Monte Carlo, conforme dito anteriormente. Para o tratamento dos dados a fim de obter-se a simulação, utilizou-se o complemento estatístico como o RNG triangular (RNG.xla) no software Microsoft Excel. A fórmula apresentada pode ser utilizada para gerar aleatoriamente um dos números inteiros $\mathrm{a}+1, \mathrm{a}+2, \ldots, \mathrm{a}+\mathrm{n}-1$ com igual probabilidade de ocorrência: RNG para a distribuição uniforme discreta: INT ( $\mathrm{n} *$ RAND ( ) ) + $\mathrm{x}$.

No estudo, o RNG foi utilizado para a distribuição uniforme discreta com o intuito de gerar números aleatórios a partir de várias outras distribuições de probabilidades.

Para a construção do simulador, foi levado em conta o tempo da atividade conforme o planejado, e as variações como tempo máximo e tempo mínimo, que podem ocorrer em cada atividade de acordo com o engenheiro responsável pela obra.

As atividades foram replicadas em amostra de 500 números, sendo assim os resultados foram obtidos com a média desses resultados.

\begin{tabular}{cccc}
\hline Atividade & $\begin{array}{c}\text { Tempo- } \\
\text { Mínimo (meses) }\end{array}$ & $\begin{array}{c}\text { Tempo- } \\
\text { estimado (meses) }\end{array}$ & $\begin{array}{c}\text { Tempo- } \\
\text { Máximo (meses) }\end{array}$ \\
\hline A & 11,5 & 12 & 12,5 \\
B & 1,5 & 2 & 2,5 \\
C & 1,5 & 2 & 2 \\
D & 3 & 4 & 4,5 \\
E & 5,5 & 6 & 5,5 \\
F & 7,5 & 8 & 8,5 \\
F1 & 1 & 1,5 & 1,5 \\
F2 & 1 & 1,5 & 1,5 \\
G1 & 1 & 1,5 & 2 \\
\hline
\end{tabular}




\begin{tabular}{llll}
\hline G2 & 1 & 1,5 & 2 \\
H & 2,5 & 3 & 3 \\
I & 2,5 & 3 & 3 \\
\hline
\end{tabular}

Tabela 4 - Possíveis durações das atividades

A tabela 5 mostra os resultados obtidos no simulador, o qual gerou números aleatórios que permitem estimar o tempo que o projeto pode levar. Foram realizadas cerca de 500 amostras, depois foi retirada a média.

\begin{tabular}{|l|l|l|l|l|l|l|}
\hline & & BCF1D & CF2G1HD & CF2G1G2ID & CEF1D & F \\
\hline Media & 12.1 & 13.4 & 12.0 & 13.1 & 13.4 & 3.9 \\
\hline DesvPad & 0.1 & 0.7 & 0.8 & 1.0 & 0.8 & 1.0 \\
\hline
\end{tabular}

Tabela 5 - Resultados médios a simulação referentes ao tempo de simulação

O tempo total de duração fornece uma média de 13.89 meses par a duração do projeto com um desvio padrão de 0.69 .

Após a simulação dos tempos de cada atividade, foi feita a simulação dos tempos em relação ao custo apresentado no cronograma e o valor estipulado para as diferentes atividades. A intenção dessa simulação é verificar o quanto varia o custo de acordo com o tempo de cada atividade e seu total. Utilizou-se o simulador dos tempos de cada atividade, multiplicando o valor de cada atividade. Com isso gerou-se o custo total de todas as atividades por mês, conforme mostrado na Figura 6.

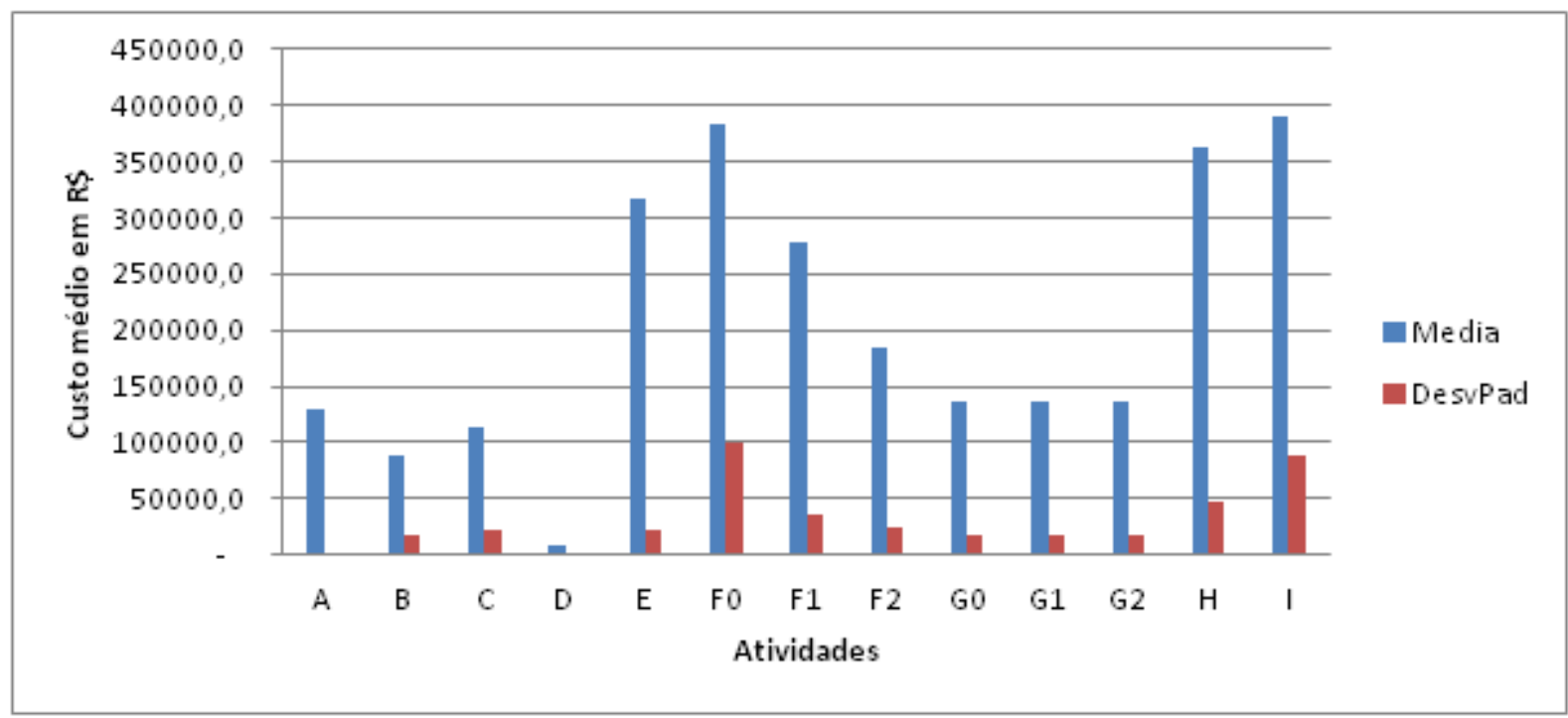

Figura 6 - Resultados dos custos

\section{CENÁRIOS}

Com o simulador criado, torna-se possível estimar possíveis alterações no decorrer da construção da rodovia, os chamados cenários. Ao alterar um determinado valor de uma das variáveis pode-se observar o comportamento do conjunto. No gráfico da Figura 7 e na Tabela 5 , pode-se verificar os tempos de acordo com o cenário proposto.

\begin{tabular}{clll}
\hline Atividade & \multicolumn{1}{c}{$\begin{array}{c}\text { Tempos cenário } 1 \\
(\text { meses })\end{array}$} & \multicolumn{1}{c}{$\begin{array}{c}\text { Tempos cenário } 2 \\
(\text { meses })\end{array}$} & $\begin{array}{c}\text { Tempos cenário 3 } \\
(\text { meses) }\end{array}$ \\
\hline A & $(11.51212 .5)$ & $(11.51212 .5)$ & $(11.51212 .5)$ \\
B & $(1.522 .5)$ & $(1.522 .5)$ & $(1.522,5)$ \\
C & $(1,522.5)$ & $(1.544)$ & $(1.51 .52)$ \\
D & $(3.565)$ & $(366)$ & $(3323.5)$ \\
E & $(1.522 .5)$ & $(5.588)$ & $(5.55 .56)$ \\
F & $(7.5912)$ & $(7.51012)$ & $(7.57 .58)$ \\
\hline
\end{tabular}




\begin{tabular}{|c|c|c|c|}
\hline F1 & (1 2.55$)$ & (1 3.5 3 3.5$)$ & $\left(\begin{array}{lll}1 & 1 & 1.5\end{array}\right)$ \\
\hline $\mathrm{F} 2$ & (1 2.55$)$ & $\left(\begin{array}{lll}1 & 3.5 & 3.5\end{array}\right)$ & $\left(\begin{array}{lll}1 & 1 & 1.5\end{array}\right)$ \\
\hline G1 & 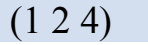 & (1 3.54$)$ & $\left(\begin{array}{lll}1 & 1 & 1.5\end{array}\right)$ \\
\hline $\mathrm{G} 2$ & $\left(\begin{array}{lll}1 & 2 & 4\end{array}\right)$ & (1 3.54$)$ & $\left(\begin{array}{lll}1 & 1 & 1.5\end{array}\right)$ \\
\hline $\mathrm{H}$ & $(2.546)$ & $(2.555)$ & $\left(\begin{array}{llll}2.5 & 2.5 & 3\end{array}\right)$ \\
\hline I & $(2.546)$ & $(2.555)$ & $\left(\begin{array}{llll}2.5 & 2.5 & 3\end{array}\right)$ \\
\hline
\end{tabular}

Tabela 5 - Durações das atividades para cada cenário

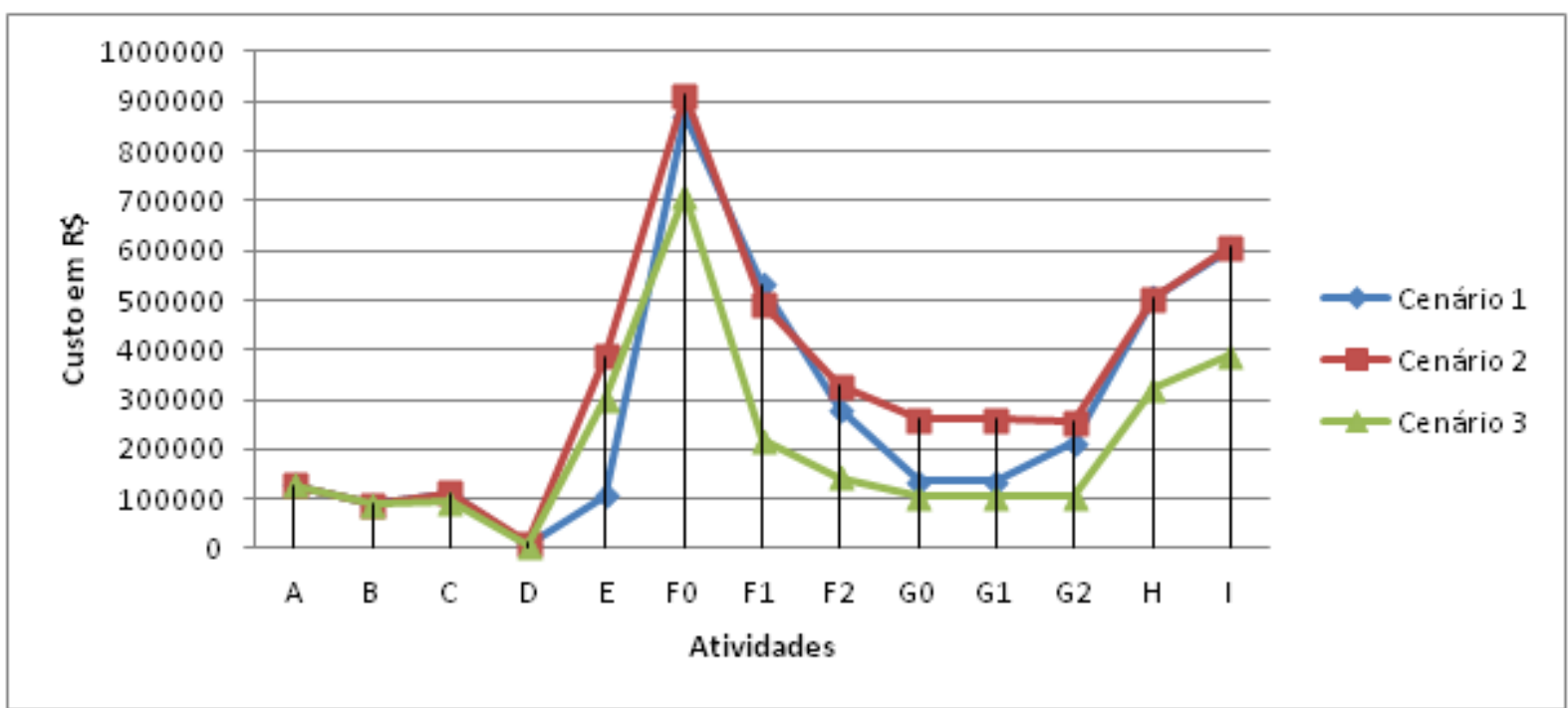

Figura 7 - Custos relacionados as atividades em cada cenário

No primeiro cenário, tem-se como simulação o aumento de tempo da atividade estrada e o impacto financeiro que causará. Atividade essa, fundamental na construção da rodovia.

A construção civil costuma sofrer com as intempéries da natureza. De acordo com o engenheiro responsável pela obra, chuvas são um dos principais motivos de ocorrer atrasos para a finalização.

Para simular um cenário no qual houve problemas com chuvas e alagamentos, aumentou-se o tempo de construção da estrada (F) previsto em 8 meses para em média 9 meses, assim como estimou-se que poderá levar até 12 meses para ser finalizada. As atividades F1, F2, D, G1, G2, H, I também foram impactadas, pois fazem parte diretamente da atividade F. Na simulação, foi alterado o tempo estimado e tempo máximo conforme pode ser verificado na tabela 5 .

O tempo médio de duração da construção da rodovia anterior aos problemas de chuva e alagamentos é de 13,41297 meses.

Ao simular os possíveis impactos previstos, a média da duração do projeto é de 18,49855 meses.

Os custos da construção também sofrem alterações, quando o projeto estava dentro do estimado, tinha o custo médio estimado em R\$ 11.155.275. Ao sofrer alteração, o custo foi para $\mathrm{R} \$ 13.906 .804$.

No segundo cenário, a hipótese trabalhada será de atraso na construção do canteiro da obra. No planejamento, o canteiro leva dois meses a ser a concluído. Por problemas no fornecimento de máquinas ou intempéries da natureza como chuvas, a instalação levará o dobro do tempo estimado, ou seja, 4 meses.

Novamente tomando por base o cenário planejado anteriormente, o tempo médio de duração da construção da rodovia de 13,41297 meses conforme mostrado no simulador.

Ao simular os possíveis impactos previstos conforme a tabela 5, a média da duração do projeto é de 20,79061 meses. 
Os custos da construção também sofrem alterações, quando o projeto estava dentro do estimado, tinha o custo médio estimado em R\$ 11.155.275. Ao sofrer alteração, o custo foi para $\mathrm{R} \$ 14.647 .620$.

No terceiro cenário, parte-se do pressuposto que a construção da rodovia tenha ficado pronta antes do planejado. Isso pode acontecer quando há rapidez na entrega dos equipamentos, repasse de verbas e ótimas condições climáticas. As alterações seguem conforme tabela 5 .

Ao representar esse cenário no simulador, pode-se observar que o tempo da duração do projeto passou a ser de 12,15885 meses, e seu custo foi de $\mathrm{R} \$ 10.559 .354$.

\section{CONCLUSÕES}

Após analisar os resultados que a rede PERT/CPM fornece, pode-se concluir que foi de extrema relevância para posteriormente simular com o método de Monte Carlo, os comportamentos das atividades, sendo assim possível visualizar quais atividades influenciam diretamente na duração e no valor da construção da rodovia.

De posse dos caminhos possíveis da construção, foi possível simular três cenários, sendo dois sujeitos a atrasos e intempéries da natureza, e um cenário otimista no qual existe um adiantamento das atividades críticas.

Os resultados mostram que atrasos em determinadas tarefas podem aumentar o tempo de duração do projeto, bem como seu custo total, podendo inclusive inviabilizar a obra. Ao haver alteração em uma atividade as demais que possuem precedência são todas impactadas, conforme tabelas 5, 6 e 7 .

De acordo com o contexto da organização e seus resultados, esta pesquisa apresenta como oportunidade de pesquisas futuras:

- Realização de estudos sobre viabilidade financeira e econômica de obras futuras, com o intuito de diminuição de custos para o orçamento público.

- Analise e aprimoramento das atividades que envolvem a logística de movimentação dos equipamentos até o canteiro onde a obra será realizada.

- Aprimoramento do cronograma físico-financeiro para que seja possível fazer o acompanhamento adequado da construção.

\section{REFERENCIAS BIBLIOGRÁFICAS}

ARAUJO, Nelma Mirian Chagas de; MEIRA, Gibson Rocha. O Papel Do Planejamento, Interligado A Um Controle Gerencial, Nas Pequenas Empresas De Construção Civil. João Pessoa, 1997.

BARRA, Renata Brabo Mascarenha; SEPTIMIO, Gabriela Andrade; BASTOS, Leonardo dos Santos; MARTINS, Vitor William. Elaboração De Rede Pert/Cpm Na Indústria Da Construção Civil Através Da Utilização Do Software Ms Project: Um Estudo De Caso. XXX III Encontro Nacional De Engenharia De Produção, Salvador, 2013.

BORBA, Rafael; NETO, Romeu; OLIVEIRA, Vinicius. A Influência Do Petróleo Na Dinâmica Econômica Das Cidades: Um Estudo Comparativo Entre Macaé (Brasil) E Aberdeen (Reino Unido). II Jornada Nacional da Produção Científica em Educação Profissional e Tecnológica, São Luis, 2007. Disponível em < http://artigocientifico.uol.com.br/uploads/artc_1199570390_37.pdf >. Acesso em 2 fev. 2014.

CUKIERMAN, Ziguimundo Salomão. Diretor da ZSC Consultoria. Aleluia! Aleluia! A Ressurreição Do PERT/CPM! Jun./2000. Disponível em $<$ http://www.zsc.com.br/ALELUIA.htm>. Acesso em 2 fev. 2014.

DONATELLI, G.D.; KONRATH, A.C. Simulação De Monte Carlo Na Avaliação De Incertezas De Medição. Revista de Ciência \& Tecnologia, v. 13, n. 25/26, p. 5-15, 2005.

GOLDSMAN, David; NANCE. Richard E; WILSON, James R. A Brief History Of Simulation. Winter Simulation Conference, 2009. 
METROPOLIS, N. The Beginning Of The Monte Carlo Method. The Alamos Science. 1987. MIRANDA, Caroline Maria Guerra; ALMEIDA, Adiel Teixeira. Seleção De Atividades Críticas Em Projetos Com Avaliação Multicritério XXXIV Simpósio Brasileiro de Pesquisa Operacional, Rio de Janeiro, 2002. Disponível em < http://www.din.uem.br/sbpo/sbpo2002/pdf/arq0085.pdf>. Acesso em 2 fev. 2014.

PIZZOLATO, Morgana. Uso De Planilhas Eletrônicas Para Implementação Do Método De Monte Carlo Para Estimativa Da Incerteza De Medição. ENQUALAB-2005 - Encontro para a Qualidade de Laboratórios, São Paulo, 2005. Disponível em < http://www.hdutil.com.br/site/arquivos/manutencao/metrologia/Uso $\% 20 \mathrm{de} \% 20$ planilias $\% 2$ 0eletronicas $\% 20$ para $\% 20$ implementacao $\% 20 \mathrm{do} \% 20$ metodo $\% 20 \mathrm{de} \% 20$ Monte $\% 20$ Carlo.pd f>. Acesso em 2 fev. 2014.

RAYCHAUDHURI, Samik. Introduction To Monte Carlo Simulation. Winter Simulation Conference, Broomfield, 2008.

RODRIGUES, Vânia Veiga; SOARES, Carlos Alberto Pereira. Metodologia Para Aplicação Da Simulação De Monte Carlo No Gerenciamento De Custos De Projetos De Construção. XII SIMPEP, Bauru, 2005.

STONNER, Rodolfo. Ferramentas De Planejamento: Utilizando O Ms Project Para Gerenciar Empreendimentos. $1^{a}$ edição. Brasil: E-Papers, 2000. 